\title{
Cardiovascular Disease in Women
}

\author{
Karla Kurrelmeyer, M.D. \\ HOUSTON METHODIST DEBAKEY HEART \& VASCULAR CENTER, HOUSTON METHODIST HOSPITAL, HOUSTON, TEXAS
}

Cardiovascular disease (CVD) is the leading cause of death among women in the United States. According to the American Heart Association's recently released 2017 update on heart disease and stroke statistics, CVD claimed 399,028 female lives in 2014 , which is more lives than those lost to all types of cancer and chronic lung disease combined. Data from the National Health and Nutrition Examination Survey (NHANES) indicates that approximately half of the 27.6 million American adults living with CVD are women. The majority (more than 8 million) of these women have ischemic heart disease (IHD), which includes both obstructive and nonobstructive coronary artery disease and acute coronary syndromes. In addition, a significant number of women are living with heart failure ( $>3$ million), pulmonary hypertension ( $>3$ million), and congenital heart disease (0.3-0.6 million). In this issue of the Methodist DeBakey Cardiovascular Journal, a group of experts from the Methodist DeBakey Heart \& Vascular Center, the Texas Medical Center, the University of Michigan, and Mayo Clinic in Jacksonville, Florida, discuss how different types of CVD affect women after menarche, during pregnancy, and after menopause and address appropriate diagnostic and therapeutic options for these conditions.

This issue opens with a review on primary and secondary prevention of CVD by Dr. Vijay Nambi, who takes an in-depth look at both traditional and nontraditional risk factors for the development of CVD in women. Dr. Nambi also discusses epidemiology and treatment of the modifiable risk factors based on the results of randomized, multicenter research trials and national guidelines. $A$ thorough understanding of CVD prevention is of paramount importance to reduce cardiovascular events, cardiovascular mortality, and the overall burden of CVD on society.

Next, Dr. Mohammed Chamsi-Pasha and I discuss the different noninvasive modalities used in the diagnosis, prognosis, and management of symptomatic women suspected of having IHD. Special attention is paid to the accuracy, prognosis, and limitations of exercise electrocardiography and stress echocardiography, and myocardial perfusion imaging, positron emission tomography, magnetic resonance imaging, and coronary computed tomography angiography. Our hope is that this information will assist clinicians in selecting the most effective noninvasive test for their symptomatic female patients.

Drs. Claire Duvernoy and Emily Perdoncin follow with a review on medical therapies and invasive treatments for women with
IHD. Registries have demonstrated that women with IHD are much less likely to receive medical therapies such as aspirin, angiotensin receptor blockers, $\beta$-blockers, aldosterone inhibitors, and statins. Likewise, women with acute coronary syndromes receive fewer cardiac catheterizations, catheterbased interventions, and bypass surgeries and are less likely to receive fibrinolytic therapy. Not surprisingly, the women in these studies demonstrated higher morbidity and mortality compared to men. Women with high-risk features showed comparable benefit from an early invasive strategy but experienced significantly more bleeding and femoral artery complications. The authors propose that dose adjustments of antithrombotic and antiplatelet therapies based on body size as well as smaller sheaths and radial artery access may reduce bleeding and vascular access complications.

This issue then explores the impact of cardiovascular disease on pregnancy. Dr. Karolina Adam first describes the normal physiologic changes that occur during pregnancy, then delves into the care of pregnant patients with common cardiac diseases such as hypertensive heart disease, valvular heart disease, cardiomyopathy, and disorders of the aorta. It is clear from her review that a team of dedicated physiciansincluding a cardiologist, maternal-fetal medicine specialist, and neonatologist-is essential for the survival of both mother and fetus. It is also clear that this is becoming a more prevalent problem for cardiologists, predominantly because the mean childbearing age for American women has shifted from the early 20 s to the 30 s and 40 s.

We move through the life cycle with a review by Drs. Biykem Bozkurt and Shaden Khalaf, who provide a comprehensive review of the epidemiology, pathophysiology, diagnosis, and prognosis of heart failure in women. Heart failure with preserved ejection fraction (HFpEF) is specifically highlighted since it is more common in women and accounts for nearly half of all hospitalizations for heart failure. Although mortality is slightly better for patients with HFpEF, readmission rates are comparable to patients with heart failure with reduced ejection fraction (HFrEF). Hypertension is the greatest risk factor for developing HFpEF and is also the single largest risk factor for cardiovascular mortality in the United States. In patients with $\mathrm{HFrEF}$, standard medical therapy, cardiac resynchronization, and left ventricular assist device placement comparably reduce morbidity and mortality for both men and women, but women have an increased risk of death 1 year following heart 
transplantation. The authors therefore conclude that further studies are needed to develop targeted therapies in women.

Drs. Myung Park and Humna Abid Memon follow with a thorough review of pulmonary hypertension (PHT), specifically focusing on the classification, gender-based epidemiologic and prognostic differences, pathophysiology, diagnosis, and treatment. Registries have demonstrated that PHT predominantly affects premenopausal women, and it has been hypothesized that this predominance occurs secondary to hormonal effects such as estrogen-induced proliferation of smooth muscle cells in the pulmonary arteries. Registries have also demonstrated that women with PHT have better long-term survival than men with PHT. However, the authors note that mortality in pregnant patients with $\mathrm{PAH}$ remains high and stress the importance of contraception and pregnancy counseling for women with PHT.

Finally, Drs. Sabrina Phillips and Michael Pirics provide information for general cardiologists who treat women with congenital heart disease. With improvements in surgical, interventional, and electrophysiology techniques, more women with congenital heart disease are surviving to childbearing age. General cardiologists often receive the first referral from an obstetrician regarding pre-conception counseling and determination of cardiovascular risk with pregnancy. The authors examine three different risk predictor models that can be helpful in determining maternal risk with pregnancy and discuss pregnancy management and outcomes in women with different types of congenital heart disease. The review confirms that most women with congenital heart disease can have a good pregnancy outcome if they are cared for and monitored by a multidisciplinary team of fetal medicine specialists, anesthesiologists, neonatologists, and cardiologists.

We hope this current issue of the Methodist DeBakey Cardiovascular Journal provides the reader with a better understanding of how CVD presents in women and how to apply preventive, diagnostic, and therapeutic strategies to care for women with CVD throughout their adult life. We invite readers to visit the journal's website at journal.houstonmethodist.org, where they will be able to have an open $Q \& A$ discussion with the authors of this issue. 